\title{
Augmented glycaemic gap is a marker for an increased risk of post-infarct left ventricular systolic dysfunction
}

\author{
Yong Zhu' ${ }^{1}$, Kesen Liu', Shuai Meng ${ }^{2}$, Ruofei Jia² ${ }^{2}$ Xuan Lei ${ }^{1}$, Maolin Chen' ${ }^{1}$, Kaiyuan Zou ${ }^{1}$, Huagang Zhu ${ }^{1}$ \\ and Zening Jin ${ }^{1,2^{*}}$
}

\begin{abstract}
Background: Left ventricular systolic dysfunction (LVSD) occurs frequently after acute ST-segment elevation myocardial infarction (STEMI). The predisposing factors and underlying mechanism of post-infarct LVSD are not fully understood. The present study mainly investigated the correlation between glycaemic gap, a novel index of stressinduced hyperglycaemia (SIH), and post-infarct LVSD.
\end{abstract}

Methods: A total of 274 first STEMI patients were enrolled in this cross-sectional study. Transthoracic echocardiography was performed within $48 \mathrm{~h}$ after admission and at 6 months after discharge to obtain left ventricular ejection fraction (LVEF). The change in LVEF was calculated as LVEF at 6 months after discharge minus baseline LVEF. Additionally, post-infarct LVSD was defined as LVEF $\leq 50 \%$. Most importantly, glycaemic gap was calculated as admission blood glucose (ABG) minus the estimated average glucose over the previous 3 months.

Results: In patients without diabetes mellitus (DM), multivariate linear regression analysis revealed that both glycaemic gap (Beta $=-1.214,95 \% \mathrm{Cl}-1.886$ to $-0.541, \mathrm{p}<0.001)$ and $\mathrm{ABG}($ Beta $=-1.124,95 \% \mathrm{Cl}-1.795$ to $-0.453, p=0.001$ ) were associated with change in LVEF. In DM patients, only glycaemic gap was still associated with change in LVEF, although this association was not observed in univariate linear regression analysis. Regarding the association between $\mathrm{SIH}$ and post-infarct LVSD, multivariate logistic regression analysis revealed that both glycaemic gap ( $O R=1.490,95 \% \mathrm{Cl} 1.043$ to $2.129, \mathrm{p}=0.028)$ and $\mathrm{ABG}(\mathrm{OR}=1.600,95 \% \mathrm{Cl} 1.148$ to $2.229, \mathrm{p}=0.005)$ were associated with an increased risk of having post-infarct LVSD in non-DM patients. However, after multivariate adjustment in DM patients, only glycaemic gap $(\mathrm{OR}=1.399,95 \% \mathrm{Cl} 1.021$ to $1.919, \mathrm{p}=0.037)$ remained associated with an increased risk of having post-infarct LVSD. Furthermore, the predictive value of glycaemic gap for postinfarct LVSD was not inferior to ABG in non-DM patients $(p=0.499)$, and only glycaemic gap, instead of ABG, could significantly predict post-infarct LVSD in DM patients ( $A U C=0.688,95 \% \mathrm{Cl} 0.591$ to $0.774, \mathrm{p}=0.002$ ).

Conclusions: Glycaemic gap was strongly associated with a change in LVEF and an increased risk of having postinfarct LVSD in patients following STEMI. In STEMI patients with DM, glycaemic gap could provide more valuable information than ABG in identifying patients at high risk of developing post-infarct LVSD.

Keywords: Left ventricular systolic dysfunction, Change in LVEF, Stress-induced hyperglycaemia, Glycaemic gap, Admission blood glucose, ST-segment elevation myocardial infarction

*Correspondence: Jin_zening@163.com

1 Department of Cardiology, Beijing Anzhen Hospital, Capital Medical University, Beijing 100029, China

Full list of author information is available at the end of the article

\section{Background}

Despite rapid development in acute treatment and second prevention in recent years, post-infarct left ventricular systolic dysfunction (LVSD) occurs frequently 
[1, 2]. Post-infarct LVSD, which is defined as reduced left ventricular ejection fraction (LVEF), is strongly correlated with adverse cardiovascular outcomes such as heart failure (HF), re-infarction, and increased cardiovascular mortality [3-6].

The pathophysiology of post-infarct LVSD is complex and poor understood [5]. Much evidence has revealed that processes such as myocardial injury associated with infarct size, left ventricular remodelling, myocardial stress, oxidative stress, and local and systemic inflammatory responses are all involved in the pathophysiology of post-infarct LVSD [5, 7-9]. Recent studies further indicated that stress-induced hyperglycaemia (SIH) and concomitant metabolic perturbations also play an extremely important role in the initiation and progression of post-infarct LVSD [10-12].

Critical illness such as acute myocardial infarction (AMI), sepsis, and major surgeries could lead to transient hyperglycaemia, termed $\mathrm{SIH}$, through multiple potential mechanisms in patients with or without diabetes mellitus (DM) [13]. Admission blood glucose (ABG) has been mainly regarded as an index of SIH in previous studies [13]. The ABG level is impacted by both acute physiological stress and chronic baseline glycaemic levels, so it does not reflect the extent of SIH accurately in an acutely ill state $[14,15]$. Furthermore, reports by Esposito et al. and Monnier et al. suggested that acute glucose fluctuations induced by SIH could be more harmful than sustained hyperglycaemia $[16,17]$. To assess the effect of SIH more accurately, glycaemic gap and stress hyperglycaemia ratio (SHR), which eliminates the interference of chronic baseline glycaemic levels, were proposed as a novel index of SIH [18-20]. Considerable evidence have confirmed both glycaemic gap and SHR were strongly correlated with adverse cardiovascular outcome in AMI patients [15, 21, 22], but we knew little about the association between these indexes and post-infarct LVSD. Therefore, we mainly investigated the correlation between glycaemic gap and post-infarct LVSD in the present study.

\section{Methods}

\section{Study population}

In this cross-sectional study, we consecutively recruited ST-segment elevation myocardial infarction (STEMI) patients admitted to Beijing Anzhen Hospital, Capital Medical University from January 2018 to January 2019. Inclusion criteria were as follows: first STEMI diagnosed according to the European Society of Cardiology and American College of Cardiology Committee criteria $[23,24]$, emergent treatment of only the infarct-related artery by primary percutaneous coronary intervention
(PCI) within $12 \mathrm{~h}$, and other diseased vessels managed by elective PCI within 1 month if required. Exclusion criteria were defined as follows: age $<18$ years or age $>80$ years, previous history of myocardial infarction or coronary revascularization, thrombolysis prior to primary PCI, without stent implantation, serious valvular heart disease and primary cardiomyopathies, estimated glomerular filtration rate $<30 \mathrm{~mL} / \mathrm{min}$ per $1.73 \mathrm{~m}^{2}$, active inflammatory or neoplastic process on admission, chronic requirement of steroid or immunosuppressive therapy. Additionally patients with hemopathy including hemolytic anemia, sickle cell anemia, thalassemia, and megaloblastic anemia were also excluded from our present study.

The study protocol was developed in compliance with the Declaration of Helsinki, and the study was approved by the Ethics Committee of Beijing Anzhen Hospital, Capital Medical University. Before the beginning of the study, we obtained written informed consent from each patient.

\section{Therapy and management}

All enrolled patients received optimal treatment according to current guidelines for the management of acute STEMI patients [23, 24]. All the patients were prescribed a loading dose of aspirin $300 \mathrm{mg}$, clopidogrel $600 \mathrm{mg}$ or ticagrelor $180 \mathrm{mg}$, and intravenously unfractionated heparin (60-70 IU/kg, maximum $5000 \mathrm{IU})$ immediately after diagnosis of STEMI. In the cardiac catheterization laboratory, all patients received a second dose of unfractionated heparin according to weight (70$100 \mathrm{IU} / \mathrm{kg}$ ). The experienced interventional cardiologist blinded to the study protocol performed the primary PCI in compliance with current guidelines [23, 24]. After primary PCI, dual antiplatelet therapy in the form of aspirin plus ticagrelor or clopidogrel was recommended for 1 year if there were no contraindications. In addition, concomitant medications such as beta-blockers, statins, and angiotensin-converting enzyme inhibitors/ angiotensin receptor blockers (ACEIs/ARBs) were all recommended unless contraindicated. Most importantly, lifestyle interventions, including smoking cessation, weight control, optimal blood pressure and blood glucose control, were also recommended.

\section{Data collection}

When patients reached the emergency department, peripheral venous blood samples were collected immediately to measure $\mathrm{ABG}$ using a standardized biochemical assay. High-performance liquid chromatography analysers were used to examine glycated haemoglobin $\left(\mathrm{HbA}_{1} \mathrm{c}\right)$ levels. Then, we obtained the estimated average glucose (eAG) level over the 
previous 3 months through the following equation: $\mathrm{eAG}=28.7 *$ HbA1c $-46.7[14,15,18]$, and glycaemic gap was calculated as ABG minus eAG. SHR, another index of relative hyperglycaemia, was defined as ABG/ eAG.

For biomarker analyses, cardiac troponin I (cTnI) and hypersensitive C-reactive protein (hs-CRP) were monitored dynamically to identify peak values. The other biological parameters, such as uric acid, homocysteine, triglyceride, low-density lipoprotein cholesterol (LDL-c), and high-density lipoprotein cholesterol (HDL-c), were all measured in a fasting state.

Demographic and clinical information, including age, sex, height, weight, smoking, DM, hypertension, and medication use at discharge, were all obtained through the standard questionnaire. In the questionnaire, DM was defined as having a prior history of DM (treated with diet or anti-diabetic medications) or having newly diagnosed DM with fasting plasma glucose $\geq 7.0 \mathrm{mmol} / \mathrm{L}$, $\mathrm{HbA}_{1} \mathrm{c} \geq 6.5 \%$ or 2 -h plasma value $\geq 11.1 \mathrm{mmol} / \mathrm{L}$ during an oral glucose tolerance test [25]. Coronary angiogram data such as number of diseased vessels (stenosis $\geq 50 \%$ of diameter of coronary artery), thrombolysis in myocardial infarction (TIMI) flow grade pre- and postPCI, and length of stents were all recorded by two independent cardiologists. Additionally, we defined slow flow/no-reflow as TIMI flow $\leq 2$ post-PCI.

\section{Echocardiography}

All patients included underwent examination of transthoracic echocardiography within $48 \mathrm{~h}$ after admission and 6 months after discharge to obtain LVEF with the modified Simpson rule. Transthoracic echocardiography was performed following recommendations from the American Society of Echocardiography [26]. Most importantly, transthoracic echocardiography was performed by the same two independent echocardiographers who have worked more than 5 years and were blinded to the study protocol with a GE Vivid 7 ultrasound machine (GE Healthcare, Piscataway, NJ, USA). Change in LVEF was defined as LVEF at 6 months post-STEMI minus baseline LVEF. Post-infarct LVSD was defined as LVEF $\leq 50 \%$.

\section{Statistics}

For continuous variables, a normal distribution was assessed using the Kolmogorov-Smirnov test. Then, continuous data were expressed as the mean \pm standard deviation or median (interquartile ranges) and compared by Student's t-test or the Mann-Whitney $U$ test. Categorical variables were summarized as numbers (percentages) and analysed by the Chi-square test. To interrogate the association between change in LVEF and other variables, multivariate linear regression analysis was implemented (age, sex, total ischaemic time, number of diseased vessels, and variables with $\mathrm{p}<0.1$ in the univariate linear regression were included in the model). Additionally, the correlation between post-infarct LVSD and other variables was determined by multivariate logistic regression analysis (the model included age, sex, and variables with $\mathrm{p}<0.1$ in the univariate logistic regression). Furthermore, we assessed the predictive value of glycaemic gap and $A B G$ through receiver operating characteristic (ROC) curve analysis. The predictive values of glycaemic gap and $A B G$ were evaluated by the area under curve (AUC), and differences in the AUC were assessed by the DeLong test. Statistical analyses were performed using SPSS 20.0 (SPSS., Chicago, IL, USA) and MedCalc V.11.4 (MedCalc, Inc., Ostend, Belgium). $\mathrm{p}<0.05$ was regarded as statistically significant.

\section{Results}

\section{Basic characteristics of the studied population}

A total of 274 STEMI patients were enrolled for the final analyses in the present study after excluding 5 patients who died within 6 months after primary PCI and 3 patients who did not attend the 6-month visit. The patients enrolled were divided into 2 subgroups (non-DM group and DM group). Then, the non-DM $(0.995 \mathrm{mmol} / \mathrm{L})$ and $\mathrm{DM}(2.427 \mathrm{mmol} / \mathrm{L})$ groups were further divided according to the median glycaemic gap level.

Table 1 shows that the mean age of the patients recruited was 57.78 years, and the prevalence of DM, current smoking, and hypertension was $39.1 \%, 65.7 \%$, and $54.0 \%$, respectively. Furthermore, as revealed in Additional file 1: Table S1, $48.5 \%$ of the patients had culprit vessel lesions in the left anterior descending artery (LAD), and $60.2 \%$ of the patients had multivessel disease. Additionally, as demonstrated in Additional file 1: Table S1, 94.5\% of patients achieved TIMI flow grade 3 after intervention, which means that almost all patients recruited received timely and successful primary PCI.

After subgroup analysis, we discovered that STEMI patients with high glycaemic gaps in the non-DM (40.97 $(14.26,75.00)$ vs. $22.35(10.20,48.77), \mathrm{p}=0.019)$ and $\mathrm{DM}$ $(47.47 \pm 31.88$ vs. $33.38 \pm 30.59, \mathrm{p}=0.022)$ groups both tended to have higher peak cTnI levels. High glycaemic gaps in non-DM $(8.66 \pm 1.47$ vs. $6.59 \pm 0.64, \mathrm{p}<0.001)$ and DM $\quad(13.88 \pm 2.95$ vs. $10.03 \pm 2.49, \quad \mathrm{p}<0.001)$ patients were also associated with higher ABG levels. Additionally, high glycaemic gap in DM patients was also associated with higher triglyceride $(2.48 \pm 2.08$ vs. $1.76 \pm 0.93, \mathrm{p}=0.022)$ levels, but not in non-DM patients. 
Table 1 Baseline characteristics

\begin{tabular}{|c|c|c|c|c|c|c|c|}
\hline \multirow[t]{2}{*}{ Clinical information } & \multirow[t]{2}{*}{ Total (274) } & \multicolumn{2}{|l|}{ Non-DM, n= 167} & \multirow[t]{2}{*}{ p-value } & \multicolumn{2}{|l|}{$\mathrm{DM}, \mathrm{n}=107$} & \multirow[t]{2}{*}{ p-value } \\
\hline & & Group 1 (84) & Group 2 (83) & & Group 3 (54) & Group 4 (53) & \\
\hline Age (years) & $57.78 \pm 11.42$ & $55.18 \pm 12.49$ & $58.19 \pm 9.95$ & 0.087 & $58.54 \pm 10.60$ & $60.49 \pm 12.08$ & 0.376 \\
\hline Male & $236(86.1 \%)$ & $77(91.7 \%)$ & $70(84.3 \%)$ & 0.160 & $44(81.5 \%)$ & $45(84.9 \%)$ & 0.797 \\
\hline BMI $\left(\mathrm{kg} / \mathrm{m}^{2}\right)$ & $25.29 \pm 2.62$ & $25.34 \pm 2.20$ & $25.22 \pm 2.84$ & 0.754 & $25.51 \pm 2.58$ & $25.10 \pm 2.94$ & 0.441 \\
\hline Smoker & $180(65.7 \%)$ & $59(70.2 \%)$ & $53(63.9 \%)$ & 0.413 & $33(61.1 \%)$ & $35(66.0 \%)$ & 0.689 \\
\hline DM & $107(39.1 \%)$ & - & - & - & $54(100.0 \%)$ & $53(100.0 \%)$ & 1.000 \\
\hline Hypertension & $148(54.0 \%)$ & $48(57.1 \%)$ & $40(48.2 \%)$ & 0.280 & $29(53.7 \%)$ & $31(58.5 \%)$ & 0.698 \\
\hline Killip classification & & & & 0.245 & & & 0.309 \\
\hline Class I & 255 (93.1\%) & 75 (89.3\%) & $80(96.4 \%)$ & & $52(96.3 \%)$ & $48(90.6 \%)$ & \\
\hline Class II & $13(4.7 \%)$ & $6(7.1 \%)$ & $2(2.4 \%)$ & & $2(3.7 \%)$ & $3(5.7 \%)$ & \\
\hline Class III & $2(0.7 \%)$ & $1(1.2 \%)$ & $1(1.2 \%)$ & & - & - & \\
\hline Class IV & $4(1.5 \%)$ & $2(2.4 \%)$ & 0 & & 0 & $2(3.8 \%)$ & \\
\hline \multicolumn{8}{|l|}{ Laboratory examination } \\
\hline Uric acid $(\mu \mathrm{mol} / \mathrm{L})$ & $359.70 \pm 86.43$ & $377.26 \pm 91.46$ & $356.86 \pm 79.79$ & 0.126 & $338.67 \pm 95.03$ & $357.74 \pm 75.17$ & 0.252 \\
\hline Homocysteine $(\mu \mathrm{mol} / \mathrm{L})$ & $12.50(9.38,17.43)$ & $14.25(10.00,23.35)$ & $12.90(9.30,18.10)$ & 0.133 & $11.05(9.18,15.25)$ & $12.30(9.15,16.60)$ & 0.413 \\
\hline $\mathrm{eGFR}(\mathrm{mmol} / \mathrm{L})$ & $99.56(91.58,107.78)$ & $99.44 \pm 15.01$ & $98.19 \pm 13.19$ & 0.571 & $99.70 \pm 14.06$ & $94.18 \pm 17.80$ & 0.077 \\
\hline $\operatorname{Tg}(\mathrm{mmol} / \mathrm{L})$ & $1.45(1.01,2.05)$ & $1.52(1.06,2.03)$ & $1.29(0.78,1.70)$ & 0.016 & $1.76 \pm 0.93$ & $2.48 \pm 2.08$ & 0.022 \\
\hline LDL-C (mmol/L) & $3.14 \pm 0.98$ & $3.26 \pm 1.05$ & $3.19 \pm 0.93$ & 0.555 & $2.95 \pm 0.83$ & $3.07 \pm 1.08$ & 0.517 \\
\hline $\mathrm{HDL}-\mathrm{C}(\mathrm{mmol} / \mathrm{L})$ & $1.05(0.88,1.20)$ & $1.07 \pm 0.25$ & $1.13 \pm 0.28$ & 0.156 & $0.98 \pm 0.21$ & $1.06 \pm 0.31$ & 0.094 \\
\hline Peak cTnl (ng/mL) & $30.80(12.40,68.52)$ & $22.35(10.20,48.77)$ & $40.97(14.26,75.00)$ & 0.019 & $33.38 \pm 30.59$ & $47.47 \pm 31.88$ & 0.022 \\
\hline Peak hs-CRP (mg/L) & $4.75(2.27,10.09)$ & $4.72(2.50,7.58)$ & $4.93(1.37,11.05)$ & 0.804 & $4.53(2.25,7.98)$ & $5.29(2.86,15.36)$ & 0.323 \\
\hline $\mathrm{ABG}(\mathrm{mmol} / \mathrm{L})$ & $8.35(7.02,10.84)$ & $6.59 \pm 0.64$ & $8.66 \pm 1.47$ & $<0.001$ & $10.03 \pm 2.49$ & $13.88 \pm 2.95$ & $<0.001$ \\
\hline $\mathrm{HBA}_{1} \mathrm{C}(\%)$ & $6.00(5.58,6.90)$ & $5.71 \pm 0.35$ & $5.62 \pm 0.40$ & 0.139 & $7.64 \pm 1.56$ & $7.48 \pm 1.31$ & 0.556 \\
\hline \multicolumn{8}{|l|}{ Pre-hospital medications } \\
\hline Insulin & $11(4.0 \%)$ & - & - & & $4(7.4 \%)$ & $7(13.2 \%)$ & 0.359 \\
\hline DPP-4 inhibitors & $22(8.9 \%)$ & - & - & & $9(16.7 \%)$ & $13(24.5 \%)$ & 0.347 \\
\hline $\begin{array}{l}\text { Other hypoglycemic } \\
\text { agents }\end{array}$ & $62(22.7 \%)$ & - & - & & $34(63.0 \%)$ & $28(52.8 \%)$ & 0.331 \\
\hline \multicolumn{8}{|l|}{ Medications at discharge } \\
\hline Aspirin & $274(100.0 \%)$ & $84(100.0 \%)$ & $83(100.0 \%)$ & 1.000 & $54(100.0 \%)$ & $53(100.0 \%)$ & 1.000 \\
\hline Clopidogrel/ticagrelor & $274(100.0 \%)$ & $84(100.0 \%)$ & $83(100.0 \%)$ & 1.000 & $54(100.0 \%)$ & $53(100.0 \%)$ & 1.000 \\
\hline Statin & $270(98.5 \%)$ & $84(100.0 \%)$ & $80(96.4 \%)$ & 0.121 & $54(100.0 \%)$ & $52(98.1 \%)$ & 0.495 \\
\hline Beta-blockers & $215(78.5 \%)$ & $68(81.0 \%)$ & $61(73.5 \%)$ & 0.273 & $45(83.3 \%)$ & $41(77.4 \%)$ & 0.474 \\
\hline ACEI/ARB & $175(63.9 \%)$ & $52(61.9 \%)$ & $48(57.8 \%)$ & 0.637 & 38 (70.4\%) & 37 (69.8\%) & 1.000 \\
\hline
\end{tabular}

Non-DM and DM patients were further divided into subgroups according to median value of glycemic gap (Group 1: glycemic gap $\leq 0.995 \mathrm{mmol} / \mathrm{L} ; \mathrm{Group} \mathrm{2:} \mathrm{glycemic}$ gap $>0.995 \mathrm{mmol} / \mathrm{L}$; Group 3: glycemic gap $\leq 2.427 \mathrm{mmol} / \mathrm{L}$; Group 4: glycemic gap $>2.427 \mathrm{mmol} / \mathrm{L}$ )

$D M$ diabetes mellitus, $B M I$ body mass index, eGFR estimated glomerular filtration rate, $T g$ triglycerides, $L D L-C$ low-density lipoprotein cholesterol, $H D L-C$ high-density lipoprotein cholesterol, $C T$ I l cardiac troponin I, $h s$ - $C R P$ hypersensitive $C$-reactive protein, $A B G$ admission blood glucose, $F B G$ fasting blood glucose, $H B A_{1} C$ glycated hemoglobin, DPP-4 inhibitors dipeptidyl peptidase-4 inhibitors, $A C E I / A R B$ angiotensin-converting enzyme inhibitor/angiotensin receptor blocker

\section{Glycaemic gap associated with change in LVEF}

In non-DM patients, compared with the low glycaemic gap group, the high glycaemic gap group was associated with lower LVEF at 6 months post-STEMI $(56.96 \pm 9.12$ vs. $60.55 \pm 6.38, \mathrm{p}=0.004$, Fig. 1a), and the change in LVEF $(4.29 \pm 6.82$ vs. $7.50 \pm 8.33, \mathrm{p}=0.007$, Fig. 1b) was also significantly smaller in the high glycaemic gap group patients, despite the baseline LVEF $(52.67 \pm 7.71$ vs. $53.11 \pm 8.65, \mathrm{p}=0.734$ ) being similar. Additionally, as presented in Fig. 1c, d, both the LVEF at the 6-month follow-up $(57.06 \pm 9.11$ vs. $60.45 \pm 6.44, \quad \mathrm{p}=0.006)$ and the change in LVEF ( $4.69 \pm 6.87$ vs. $7.11 \pm 8.42$, $\mathrm{p}=0.044$ ) were significantly lower in the high $A B G$ group than in the low $A B G$ group in non-DM patients. Most importantly, as shown in Table 2, glycaemic gap $($ Beta $=-1.214,95 \% \mathrm{CI}-1.886$ to $-0.541, \mathrm{p}<0.001)$ and ABG (Beta $=-1.124,95 \%$ CI -1.795 to -0.453 , $\mathrm{p}=0.001$ ) remained associated with the change in LVEF 

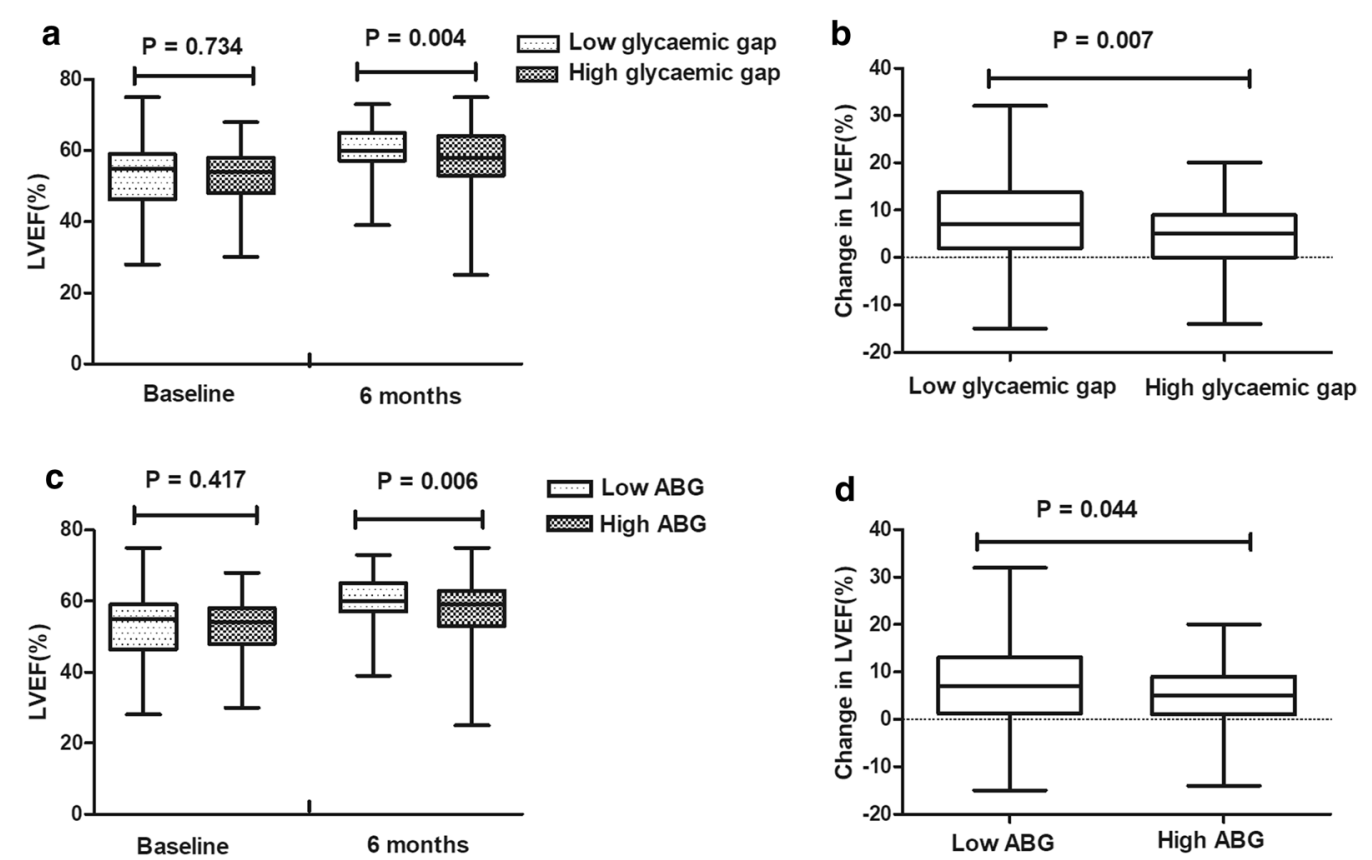

Fig. 1 LVEF (a) and change in LVEF (b) in non-DM patients in the high glycaemic gap group and low glycaemic gap group at 6-month follow-up. LVEF (c) and change in LVEF (d) in non-DM patients in the high ABG group and low ABG group at 6-month follow-up. LVEF left ventricular ejection fraction, DM diabetes mellitus, ABG admission blood glucose. Change in LVEF was defined as LVEF at 6 months post-STEMI minus baseline LVEF

Table 2 Multivariate linear regression for the correlation between change in LVEF and other variables in non-DM

\begin{tabular}{|c|c|c|c|c|c|c|c|c|c|}
\hline \multirow{2}{*}{$\begin{array}{l}\text { Non-DM patients } \\
\text { Variables }\end{array}$} & \multicolumn{3}{|c|}{ Univariate linear regression } & \multicolumn{3}{|c|}{$\begin{array}{l}\text { Multivariate linear regression (Mode } \\
A, R^{2}=0.379 \text { ) }\end{array}$} & \multicolumn{3}{|c|}{$\begin{array}{l}\text { Multivariate linear regression (Mode } \\
B, R^{2}=0.373 \text { ) }\end{array}$} \\
\hline & Beta & $95 \% \mathrm{Cl}$ & p-value & Beta & $95 \% \mathrm{Cl}$ & p-value & Beta & $95 \% \mathrm{Cl}$ & $\mathrm{p}$-value \\
\hline Age & -0.029 & -0.134 to 0.076 & 0.582 & 0.077 & -0.015 to 0.169 & 0.099 & 0.098 & 0.004 to 0.193 & 0.042 \\
\hline Sex & -3.811 & -7.428 to -0.193 & 0.039 & -4.107 & -7.250 to -0.964 & 0.011 & -4.337 & -7.490 to -1.183 & 0.007 \\
\hline BMI & 0.463 & -0.003 to 0.928 & 0.051 & 0.286 & -0.109 to 0.680 & 0.155 & 0.318 & -0.078 to 0.714 & 0.115 \\
\hline Peak hs-CRP & -0.152 & -0.304 to 0.001 & 0.051 & -0.185 & -0.312 to -0.058 & 0.005 & -0.190 & -0.318 to -0.063 & 0.004 \\
\hline Baseline LVEF & -0.469 & -0.596 to -0.342 & $<0.001$ & -0.492 & -0.614 to -0.370 & $<0.001$ & -0.498 & -0.621 to -0.375 & $<0.001$ \\
\hline Total ischemic time & -0.004 & -0.012 to 0.004 & 0.318 & -0.005 & -0.011 to 0.001 & 0.114 & -0.005 & -0.011 to 0.002 & 0.148 \\
\hline No. of diseased vessels & -0.516 & -1.958 to 0.927 & 0.481 & -0.171 & -1.364 to 1.022 & 0.778 & -0.174 & -1.374 to 1.026 & 0.775 \\
\hline $\mathrm{ABG}$ & -0.866 & -1.634 to -0.099 & 0.027 & & & & -1.124 & -1.795 to -0.453 & 0.001 \\
\hline Glycemic gap & -1.116 & -1.901 to -0.331 & 0.006 & -1.214 & -1.886 to -0.541 & $<0.001$ & & & \\
\hline
\end{tabular}

Change in LVEF was defined as LVEF at 6-month follow-up minus baseline LVEF

$D M$ diabetes mellitus, $B M I$ body mass index, $h s$-CRP hypersensitive C-reactive protein, $L V E F$ left ventricular ejection fraction, Total ischemic time the period from symptom onset to reopening of infarction-associated artery, $A B G$ admission blood glucose

in non-DM patients after adjusting for age, sex, BMI, peak hs-CRP, baseline LVEF, total ischaemic time, and number of diseased vessels.

As demonstrated in Fig. 2a, b, a high glycaemic gap was still associated with lower LVEF (56.13 \pm 9.38 vs. $59.61 \pm 6.28, \mathrm{p}=0.027)$ and change in LVEF $(3.43 \pm 7.80$ vs. $7.31 \pm 7.51, \mathrm{p}=0.010)$ at the 6 -month follow-up in DM patients. However, as presented in Fig. 2c, d, we observed no difference in LVEF and change in LVEF between the high ABG group and the low ABG group in DM patients at the 6-month follow-up. In DM patients, univariate linear regression revealed that both glycaemic gap (Beta $=-0.366,95 \% \mathrm{CI}-0.942$ to $0.210, \mathrm{p}=0.210$ ) and $\mathrm{ABG}$ (Beta $=-0.281,95 \% \mathrm{CI}-0.734$ to 0.172 , $\mathrm{p}=0.221$ ) were not correlated with change in LVEF. 

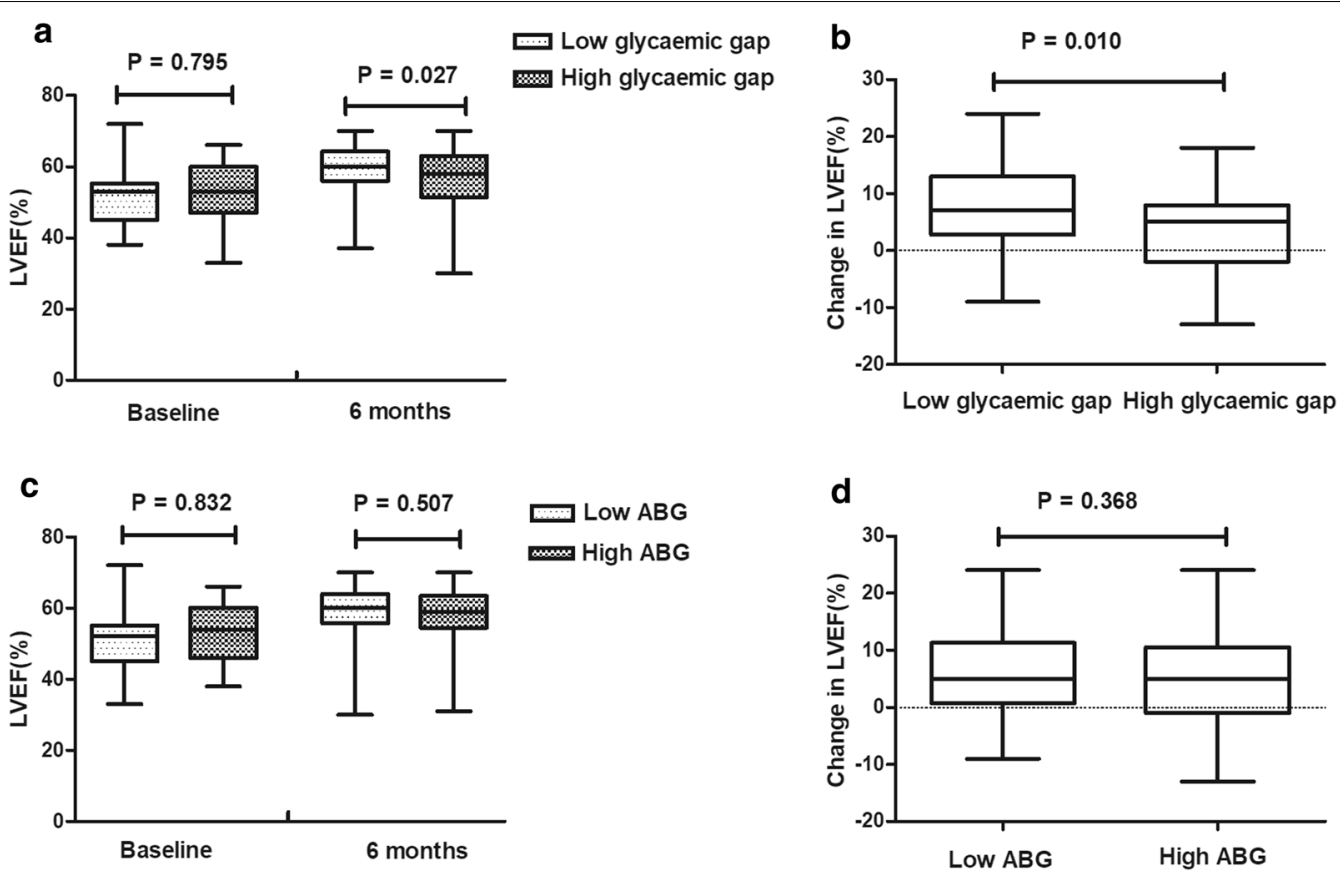

Fig. 2 LVEF (a) and change in LVEF (b) in DM patients in the high glycaemic gap group and low glycaemic gap group at 6-month follow-up. LVEF (c) and change in LVEF (d) in DM patients in the high ABG group and low ABG group at 6-month follow-up. LVEF left ventricular ejection fraction, DM diabetes mellitus, ABG admission blood glucose. Change in LVEF was defined as LVEF at 6 months post-STEMI minus baseline LVEF

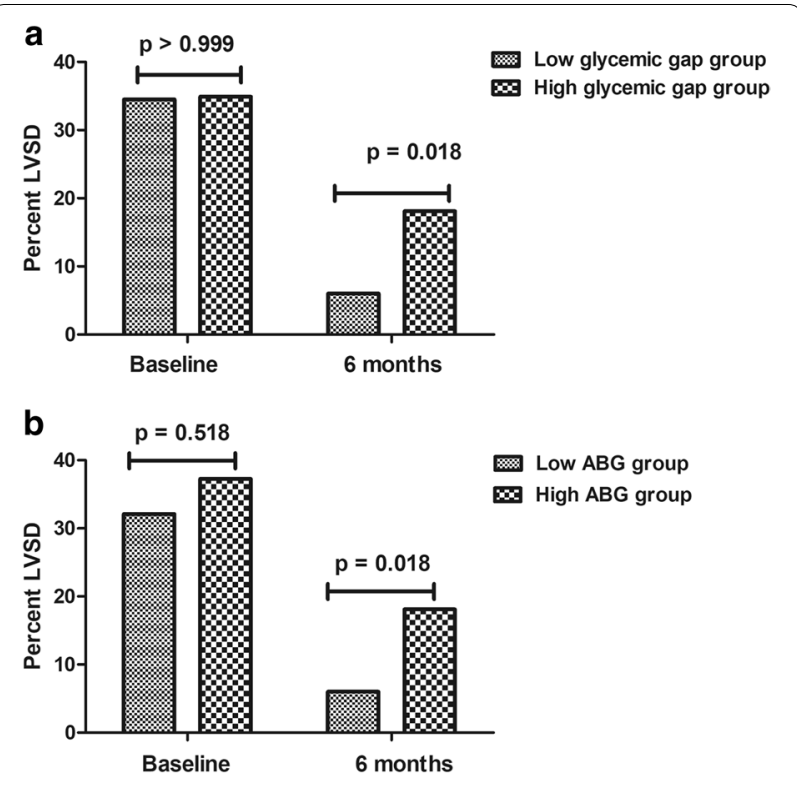

Fig. 3 The proportion of post-infarct LVSD in non-DM patients in the high glycaemic gap group and low glycaemic gap group at baseline and 6-month follow-up (a). The proportion of post-infarct LVSD in non-DM patients in the high ABG group and low ABG group at baseline and 6-month follow-up (b). LVSD left ventricular systolic dysfunction, DM diabetes mellitus, $A B G$ admission blood glucose. Post-infarct LVSD was defined as LVEF $\leq 50 \%$ after infarction

\section{Glycaemic gap was strongly correlated with post-infarct LVSD}

In non-DM patients, the proportion of post-infarct LVSD in high glycaemic gap patients was significantly higher than that in low glycaemic gap patients at the 6-month follow-up $(18.1 \%$ vs. $6.0 \%, p=0.018$, Fig. 3a), although the proportion of post-infarct LVSD was similar between subgroups at baseline (34.9\% vs. 34.5\%, p >0.99, Fig. 3a). Additionally, as revealed in Fig. 3b, post-infarct LVSD at the 6-month follow-up also occurred more often in the high ABG group patients $(18.1 \%$ vs. $6.0 \%, \mathrm{p}=0.018)$. Furthermore, as revealed in Table 3, multivariate logistic regression revealed that an augmented glycaemic gap $(\mathrm{OR}=1.490,95 \% \mathrm{CI} 1.043$ to $2.129, \mathrm{p}=0.028)$ and $\mathrm{ABG}$ $(\mathrm{OR}=1.600,95 \% \mathrm{CI} 1.148$ to $2.229, \mathrm{p}=0.005)$ were associated with an increased risk of having post-infarct LVSD after adjusting for age, sex, peak cTnI, HDL-c, and peak hs-CRP in non-DM patients.

As shown in Fig. 4a, although the proportion of postinfarct LVSD at baseline was similar between subgroups in DM patients, high glycaemic gap patients were still associated with a higher proportion of post-infarct LVSD at the 6-month follow-up compared with low glycaemic gap patients $(22.6 \%$ vs. $5.6 \%, \mathrm{p}=0.013)$. In line with this finding, as indicated in Table 3, univariate logistic regression revealed that an elevated glycaemic gap was strongly associated with an increased risk of having 
Table 3 Multivariate logistic regression for the correlation between post-infarct LVSD and other variables in non-DM and DM patients

\begin{tabular}{|c|c|c|c|c|c|c|c|c|c|}
\hline \multirow{2}{*}{$\begin{array}{l}\text { Non-DM patients } \\
\text { Variables }\end{array}$} & \multicolumn{3}{|c|}{ Univariate logistic regression } & \multicolumn{3}{|c|}{$\begin{array}{l}\text { Multivariate logistic regression (Mode } \\
\text { A) }\end{array}$} & \multicolumn{3}{|c|}{$\begin{array}{l}\text { Multivariate logistic regression } \\
\text { (Mode B) }\end{array}$} \\
\hline & OR & $95 \% \mathrm{Cl}$ & p-value & OR & $95 \%$ & p-value & OR & $95 \%$ & $\mathrm{p}$-value \\
\hline Age & 0.986 & 0.947 to 1.027 & 0.494 & 0.950 & 0.897 to 1.005 & 0.073 & 0.939 & 0.884 to 0.996 & 0.038 \\
\hline Sex & 0.341 & 0.109 to 1.071 & 0.065 & 0.216 & 0.046 to 1.017 & 0.053 & 0.195 & 0.041 to 0.934 & 0.041 \\
\hline Peak cTnl & 1.025 & 1.008 to 1.042 & 0.003 & 1.015 & 0.997 to 1.033 & 0.106 & 1.015 & 0.997 to 1.034 & 0.111 \\
\hline $\mathrm{HDL}-\mathrm{C}$ & 5.707 & 1.167 to 27.919 & 0.032 & 3.972 & 0.600 to 26.284 & 0.153 & 4.626 & 0.679 to 31.509 & 0.118 \\
\hline Peak hs-CRP & 1.074 & 1.020 to 1.131 & 0.006 & 1.070 & 1.010 to 1.135 & 0.022 & 1.075 & 1.013 to 1.141 & 0.017 \\
\hline ABG & 1.499 & 1.146 to 1.962 & 0.003 & & & & 1.600 & 1.148 to 2.229 & 0.005 \\
\hline Glycemic gap & 1.494 & 1.120 to 1.993 & 0.006 & 1.490 & 1.043 to 2.129 & 0.028 & & & \\
\hline \multirow{2}{*}{$\begin{array}{l}\text { DM patients } \\
\text { Variables }\end{array}$} & \multicolumn{3}{|c|}{ Univariate logistic regression } & \multicolumn{3}{|c|}{ Multivariate logistic regression } & & & \\
\hline & OR & $95 \% \mathrm{Cl}$ & p-value & OR & $95 \% \mathrm{Cl}$ & p-value & & & \\
\hline Age & 1.028 & 0.977 to 1.081 & 0.287 & 1.013 & 0.945 to 1.085 & 0.722 & & & \\
\hline Sex & 3.038 & 0.894 to 10.326 & 0.075 & 0.317 & 0.057 to 1.780 & 0.192 & & & \\
\hline Peak cTnl & 1.048 & 1.023 to 1.073 & $<0.001$ & 1.051 & 1.023 to 1.080 & $<0.001$ & & & \\
\hline $\mathrm{HDL}-\mathrm{C}$ & 6.183 & 0.975 to 39.221 & 0.053 & 0.588 & 0.073 to 4.736 & 0.617 & & & \\
\hline Peak hs-CRP & 1.025 & 0.964 to 1.090 & 0.434 & & & & & & \\
\hline $\mathrm{ABG}$ & 1.106 & 0.947 to 1.292 & 0.203 & & & & & & \\
\hline Glycemic gap & 1.267 & 1.027 to 1.565 & 0.027 & 1.399 & 1.021 to 1.919 & 0.037 & & & \\
\hline
\end{tabular}

LVSD was defined as LVEF $\leq 50 \%$

LVSD left ventricular systolic dysfunction, DM diabetes mellitus, CTnI cardiac troponin I, HDL-C high-density lipoprotein cholesterol, hs-CRP hypersensitive C-reactive protein, $A B G$ admission blood glucose

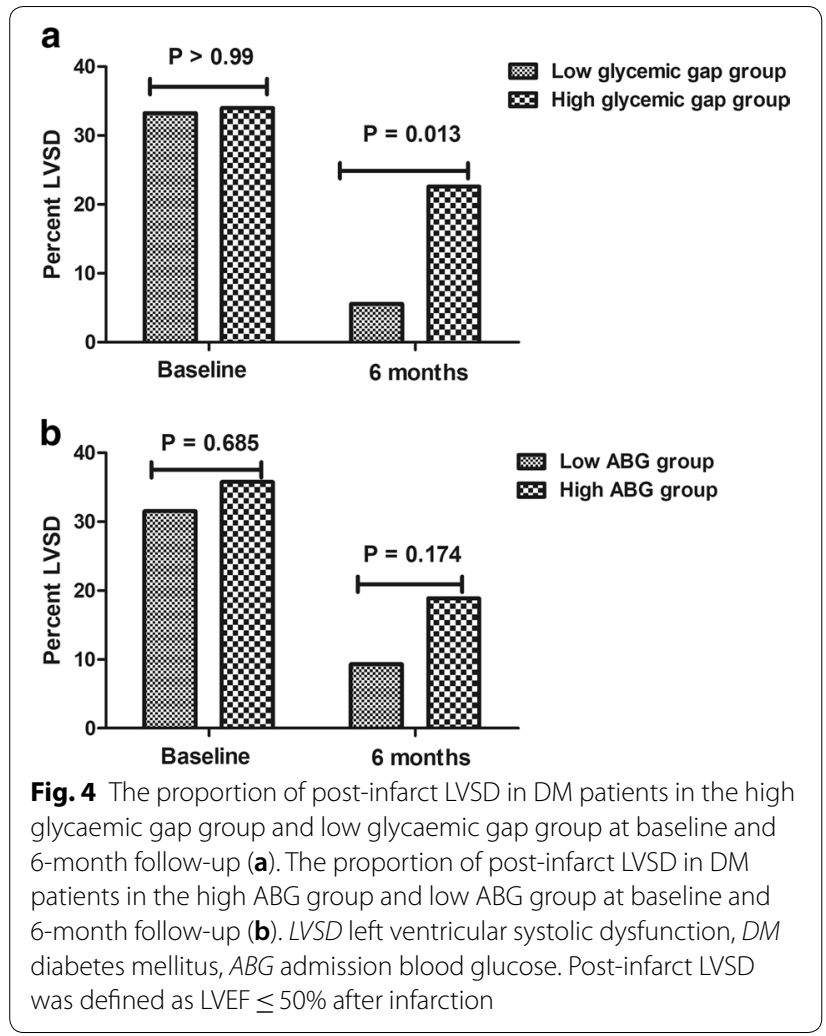

post-infarct LVSD at 6 months post-STEMI $(\mathrm{OR}=1.267$, 95\% CI 1.027 to $1.565, \mathrm{p}=0.027)$. After adjusting for age, sex, peak cTnI, and HDL-c through multivariate logistic regression, the correlation persisted in DM patients $(\mathrm{OR}=1.399,95 \%$ CI 1.021 to $1.919, \mathrm{p}=0.037)$. However, as indicated in Fig. 4b, there were no significant differences between the high ABG and low ABG groups in the prevalence of post-infarct LVSD at baseline and 6 months post-STEMI. Additionally, univariate logistic regression also revealed that $\mathrm{ABG}$ also was not correlated with post-infarct LVSD at the 6-month follow-up $(\mathrm{OR}=1.106,95 \% \mathrm{CI} 0.947$ to $1.292, \mathrm{p}=0.203)$.

\section{Predictive value of glycaemic gap compared to ABG for post-infarct LVSD at the 6-month follow-up}

After ROC analysis (Table 4), we demonstrated that both glycaemic gap (AUC $=0.697,95 \%$ CI 0.622 to 0.766 , $\mathrm{p}=0.001)$ and $\mathrm{ABG}$ (AUC $=0.713,95 \% \mathrm{CI} 0.638$ to 0.781 , $\mathrm{p}=0.002$ ) could provide a moderate predictive value for post-infarct LVSD at the 6-month follow-up, and the predictive value of glycaemic gap was not inferior to $\mathrm{ABG}$ $(\mathrm{p}=0.499)$ in non-DM patients. However, as shown in Table 4, only glycaemic gap had a moderate predictive value for post-infarct LVSD at 6 months after STEMI 
Table 4 Predictive value of glycemic gap versus ABG for post-infarct LVSD at 6-month follow-up

\begin{tabular}{|c|c|c|c|c|c|c|c|c|c|}
\hline & AUC & $95 \% \mathrm{Cl}$ & $p$ for AUC & $P$ for $\delta A U C$ & Cut-off value & Sensitivity & Specificity & PPV & NPV \\
\hline \multicolumn{10}{|c|}{ Non-DM patients } \\
\hline $\mathrm{ABG}$ & 0.713 & 0.638 to 0.781 & 0.002 & - & 8.27 & $70.00 \%$ & $78.23 \%$ & $30.4 \%$ & $95.0 \%$ \\
\hline Glycemic gap & 0.697 & 0.622 to 0.766 & 0.001 & 0.499 & 1.858 & $55.00 \%$ & $78.91 \%$ & $26.2 \%$ & $92.8 \%$ \\
\hline \multicolumn{10}{|l|}{ DM patients } \\
\hline$A B G$ & 0.621 & 0.522 to 0.713 & 0.08 & & & & & & \\
\hline Glycemic gap & 0.688 & 0.591 to 0.774 & 0.002 & & 2.197 & $86.67 \%$ & $54.35 \%$ & $23.6 \%$ & $96.2 \%$ \\
\hline
\end{tabular}

$A B G$ admission blood glucose, LVSD left ventricular systolic dysfunction, DM diabetes mellitus, $A U C$ area under curve by receiver-operating characteristic curve analysis, $\delta$ AUC difference of AUC, PPV positive predictive values, NPV negative predictive values

$(\mathrm{AUC}=0.688,95 \% \mathrm{CI} 0.591$ to $0.774, \mathrm{p}=0.002)$ in $\mathrm{DM}$ patients.

\section{Discussion}

Our present study demonstrated 3 significant findings. First, glycaemic gap, as a novel index of SIH, was associated with changes in LVEF and the increased risk of having post-infarct LVSD in STEMI patients with or without DM. Second, the correlation between change in LVEF, post-infarct LVSD, and ABG only persisted in non-DM patients. Third, in regard to identifying patients at high risk of having post-infarct LVSD, glycaemic gap could provide a superior discriminatory ability compared with ABG in DM patients.

Considerable evidences suggested glycaemic excursion is not only associated with risk of cardiovascular disease but also associated with major adverse cardiovascular and cerebrovascular events [27, 28]. Glycaemic gap, as a novel marker of glycaemic excursion that quantifies the magnitude of a relative glycaemic rise from chronic glycaemia in an acutely ill state, was proposed to better assess the effect of SIH [20]. Previous studies have reported glycaemic gap is associated with severity of disease and unfavorable prognosis in patients with critical illness [29]. Our present study further confirmed that peak cTnI was significantly higher in patients with a high glycaemic gap level regardless of diabetic status, which suggests $\mathrm{SIH}$ measured by glycaemic gap is associated with infarct size assessed by peak cTnI. According to previous studies, the potential mechanisms of the relationship between $\mathrm{SIH}$ and myocardial injury included secretion of excessive cortisol and catecholamine, inflammation and oxidative stress, endothelial dysfunction, relative insulin deficiency, and prothrombotic state $[13,16,17,30]$.

Recent clinical trials have confirmed ABG levels are associated with poor prognosis in STEMI patients without DM, but the association is relative weak in DM patients [31, 32]. However, the association between ABG and myocardial function after STEMI in patients with
DM or without DM is not well investigated at present, and direct evidence is limited. Capes et al. reported that ABG was correlated with an increased risk of congestive heart failure or cardiogenic shock in non-DM patients, but the correlation was not observed in DM patients [31]. Teraguchi et al. further demonstrated that myocardial salvage index (MSI) assessed cardiac magnetic resonance $(\mathrm{CMR})$ in patients with $\mathrm{SIH}(\mathrm{ABG} \geq 10 \mathrm{mmol} / \mathrm{L})$ was lower than that in patients without SIH among non-DM patients; however, no significant difference was observed in MSI between patients with or without SIH in DM patients [33]. In line with these studies, our present study revealed that ABG was associated with changes in LVEF and the risk of having post-infarct LVSD in non-DM patients, but the association was not observed in DM patients. There may be several explanations for the discrepant findings. First, not taking baseline glucose levels into consideration in DM patients may be the main reason. There is a wide range in the level of chronic glycaemic control among DM patients, from satisfactory to poor; therefore, it is necessary to consider baseline glucose levels when investigating the correlation between SIH and the risk of post-infarct LVSD. Second, many DM patients achieve good glycaemic control with optimal glucose-lowering treatment, while others do not.

Instead of ABG, both glycaemic gap and SHR were associated with major adverse cardiac events (MACEs) and could provide moderate predictive value for the occurrence of MACEs in AMI patients with DM [15, 22, 34]. Liao et al. further demonstrated glycaemic gap rather than ABG was linked to all-cause mortality in acute heart failure (AHF) patients with DM [35]. Moreover, they also discovered glycaemic gap could provide higher predictive value than ABG for all-cause mortality, cardiovascular mortality, and acute respiratory failure [35]. Extending prior studies, our present study demonstrated for the first time that glycaemic gap, not ABG, was associated with the change in LVEF and risk of having post-infarct LVSD in DM patients. Comparing with ABG, glycaemic gap could provide a superior predictive ability for post-infarct 
LVSD in DM patients. Therefore, glycaemic gap could be used to assess prognosis and identify patients at high risk for developing post-infarct LVSD in the management of AMI patients, particularly in patients with DM. However, we should note that the accuracy of glycaemic gap is still moderate, and its performance should be further explored by prospective longitudinal studies before putting it into practice.

Additionally, Lee et al. reported that relative hyperglycaemia during glucose-lowering treatment is more strongly associated with adverse outcomes than absolute glycaemia in patients following AMI [36]. The results suggested that using relative, not absolute, glycaemic thresholds for intervention and therapeutic glycaemic targets could improve adverse outcomes following AMI [36]. Therefore, it is necessary for future randomized controlled trials to investigate whether a glucose-lowering treatment target on glycaemic gap or SHR could reduce the risk of developing post-infarct LVSD in STEMI patients.

We acknowledge that the present study has several limitations. First, this was a single-centre study with a limited sample size. Therefore, experimental and multicenter clinical studies with large sample size are needed to further verify our findings in the future. Second, this was an observational study, which failed to assess the causal link between the relative hyperglycaemia glycaemic gap and post-infarct LVSD. Third, we evaluated changes in LVEF and post-infarct LVSD only through transthoracic echocardiography, which may limit the value of our research. To improve accuracy and provide more valuable information, future studies could employ CMR or single-photon emission computed tomography to assess associated parameters including LVEF, infarct size, microvascular obstruction, and MSI. Finally, we only recruited STEMI patients who underwent primary PCI successfully in the present study. Thus, our findings remain to be verified in general patients with acute coronary syndrome.

\section{Conclusions}

Our present study demonstrated for the first time that glycaemic gap, as a novel index of $\mathrm{SIH}$, was associated with the change in LVEF and the risk of having postinfarct LVSD at the 6-month follow-up in STEMI patients after primary PCI. Compared to ABG, glycaemic gap could provide a superior predictive value for postinfarct LVSD in DM patients and an equivalent predictive value in non-DM patients. Prospective studies are needed to further investigate whether glycaemic control using glycaemic gap could reduce the risk of having postinfarct LVSD, especially in DM patients.

\section{Supplementary information}

Supplementary information accompanies this paper at https://doi. org/10.1186/s12933-020-01075-8.

Additional file 1: Table S1. Procedural characteristics of patients enrolled.

\section{Abbreviations}

LVSD: Left ventricular systolic dysfunction; LVEF: Left ventricular ejection fraction; HF: Heart failure; SIH: Stress-induced hyperglycaemia; AMI: Acute myocardial infarction; DM: Diabetes mellitus; ABG: Admission blood glucose; SHR: Stress hyperglycaemia ratio; STEMI: ST-segment elevation myocardial infarction; PCl: Percutaneous coronary intervention; ACEls/ARBs: Angiotensinconverting enzyme inhibitors/angiotensin receptor blockers; HbA1c: Glycated haemoglobin; eAG: Estimated average glucose; cTnl: Cardiac troponin I; hs-CRP: Hypersensitive C-reactive protein; LDL-c: Low-density lipoprotein cholesterol; HDL-c: High-density lipoprotein cholesterol; TIMI: Thrombolysis in myocardial infarction; ROC: Receiver operating characteristic; AUC: Area under curve; LAD: Left anterior descending artery; MSI: Myocardial salvage index; CMR: Cardiac magnetic resonance; MACEs: Major adverse cardiac events; AHF: Acute heart failure.

\section{Acknowledgements}

None.

\section{Authors' contributions}

YZ and KSL interpreted the data and wrote the manuscript. ZNJ and HGZ designed the study protocol and supervised the project. ZNJ, HGZ, SM and RFJ performed primary $\mathrm{PCI}$. XL, MLC, and KYZ enrolled and managed the participants. All authors read and approved the final manuscript.

\section{Funding}

No.

\section{Availability of data and materials}

The datasets used and analysed during the current study are available from the corresponding author on reasonable request.

\section{Ethics approval and consent to participate}

The study protocol was developed in compliance with the Declaration of Helsinki, and the study was approved by the Ethics Committee of Beijing Anzhen Hospital, Capital Medical University. Before the beginning of the study, we obtained written informed consent from each patient.

\section{Consent for publication}

Not applicable.

\section{Competing interests}

The authors declare that they have no competing interests.

\section{Author details}

${ }^{1}$ Department of Cardiology, Beijing Anzhen Hospital, Capital Medical University, Beijing 100029, China. ${ }^{2}$ Department of Cardiology, Beijing Tiantan Hospital, Capital Medical University, Beijing, China.

Received: 2 April 2020 Accepted: 2 July 2020

Published online: 04 July 2020

\section{References}

1. Puymirat E, Simon T, Steg PG, Schiele F, Gueret P, Blanchard D, Khalife K, Goldstein P, Cattan S, Vaur L, et al. Association of changes in clinical characteristics and management with improvement in survival among patients with ST-elevation myocardial infarction. JAMA. 2012;308(10):998-1006.

2. Weir RAP, McMurray JJV, Velazquez EJ. Epidemiology of heart failure and left ventricular systolic dysfunction after acute myocardial infarction: 
prevalence, clinical characteristics, and prognostic importance. Am J Cardiol. 2006;97(10-supp-S1):13-25.

3. Kümler T, Gislason GH, Køber L, Torp-Pedersen C. Persistence of the prognostic importance of left ventricular systolic function and heart failure after myocardial infarction: 17-year follow-up of the TRACE register. Eur J Heart Fail. 2010;12(8):805-11.

4. Im MS, Kim H, Kim S, Lim W, Seo J, Chung W, Zo J, Kim M, Park K, Koo B. Different prognostic factors according to left ventricular systolic function in patients with acute myocardial infarction. Int J Cardiol. 2016:221:90-6.

5. Swiatkiewicz I, Taub PR. The usefulness of $\mathrm{C}$-reactive protein for the prediction of post-infarct left ventricular systolic dysfunction and heart failure. Kardiol Pol. 2018;76(5):821-9.

6. Swiatkiewicz I, Kozinski M, Magielski P, Gierach J, Fabiszak T, Kubica A, Sukiennik A, Navarese EP, Odrowaz-Sypniewska G, Kubica J. Usefulness of C-reactive protein as a marker of early post-infarct left ventricular systolic dysfunction. Inflamm Res. 2012;61(7):725-34.

7. van der Bijl P, Abou R, Goedemans L, Gersh BJ, Holmes DR, Ajmone Marsan N, Delgado V, Bax JJ. Left ventricular post-infarct remodeling. JACC Heart Failure. 2020;8(2):131-40.

8. Parviz Y, Vijayan S, Lavi S. A review of strategies for infarct size reduction during acute myocardial infarction. Cardiovasc Revasc Med. 2017;18(5):374-83.

9. Świątkiewicz I, Magielski P, Kubica J, Zadourian A, DeMaria AN, Taub PR. Enhanced inflammation is a marker for risk of post-infarct ventricular dysfunction and heart failure. Int J Mol Sci. 2020;21 (3):807.

10. Bauters C, Ennezat PV, Tricot O, Lauwerier B, Lallemant R, Saadouni $\mathrm{H}$, Quandalle P, Jaboureck O, Lamblin N, Le Tourneau T. Stress hyperglycaemia is an independent predictor of left ventricular remodelling after first anterior myocardial infarction in non-diabetic patients. Eur Heart J. 2006;28(5):546-52.

11. Ishihara M, Inoue I, Kawagoe T, Shimatani Y, Kurisu S, Nishioka K Umemura T, Nakamura S, Yoshida M. Impact of acute hyperglycemia on left ventricular function after reperfusion therapy in patients with a first anterior wall acute myocardial infarction. Am Heart J. 2003;146(4):674-8.

12. Yang $C D$, Shen $Y$, Lu L, Ding FH, Yang ZK, Zhang RY, Shen WF, Jin W, Wang $X Q$. Insulin resistance and dysglycemia are associated with left ventricular remodeling after myocardial infarction in non-diabetic patients. Cardiovasc Diabetol. 2019:18(1):100.

13. Chandalia H, Gokani A. Stress hyperglycaemia. Lancet. 2009:324(8406):811-2

14. Yang Y, Kim T, Yoon K, Chung WS, Ahn Y, Jeong M, Seung K, Lee S, Chang $K$. The stress hyperglycemia ratio, an index of relative hyperglycemia, as a predictor of clinical outcomes after percutaneous coronary intervention. Int J Cardiol. 2017;241:57-63.

15. Gao S, Liu Q, Ding X, Chen H, Zhao X, Li H. Predictive value of the acuteto-chronic glycemic ratio for in-hospital outcomes in patients with ST-segment elevation myocardial infarction undergoing percutaneous coronary intervention. Angiology. 2020;71(1):38-47.

16. Monnier L, Mas E, Ginet C, Michel FO, Villon L, Cristol J, Colette C. Activation of oxidative stress by acute glucose fluctuations compared with sustained chronic hyperglycemia in patients with type 2 diabetes. JAMA. 2006;295(14):1681.

17. Esposito K, Nappo F, Marfella R, Giugliano G, Giugliano F, Ciotola M, Quagliaro L, Ceriello A, Giugliano D. Inflammatory cytokine concentrations are acutely increased by hyperglycemia in humans. Circulation. 2002;106(16):2067-72.

18. Roberts GW, Quinn SJ, Valentine N, Alhawassi T, O'Dea H, Stranks SN Burt MG, Doogue MP. Relative hyperglycemia, a marker of critical illness: introducing the stress hyperglycemia ratio. J Clin Endocr Metab. 2015;100(12):4490-7.

19. Chen X, Liu Z, Miao J, Zheng W, Yang Q, Ye X, Zhuang X, Peng F. High stress hyperglycemia ratio predicts poor outcome after mechanical thrombectomy for ischemic stroke. J Stroke Cerebrovasc Dis. 2019;28(6):1668-73.

20. Chen P, Tsai S, Wang J, Tzeng Y, Wang Y, Chu C, Chu S, Liao W. An elevated glycemic gap predicts adverse outcomes in diabetic patients with necrotizing fasciitis. PLoS ONE. 2019;14(10):e223126.

21. Kojima T, Hikoso S, Nakatani D, Suna S, Dohi T, Mizuno H, Okada K, Kitamura T, Kida H, Oeun B, et al. Impact of hyperglycemia on long-term outcome in patients with ST-segment elevation myocardial infarction. Am J Cardiol. 2020;125(6):851-9.

22. Liao W, Lin C, Lee C, Wu Y, Chang W, Hsu C, Wang J, Tsai S. An elevated glycemic gap is associated with adverse outcomes in diabetic patients with acute myocardial infarction. Sci Rep. 2016;6(1):1.

23. Ibanez B, James S, Agewall S, Antunes MJ, Bucciarelli-Ducci C, Bueno H, Caforio ALP, Crea F, Goudevenos JA, Halvorsen S, et al. 2017 ESC Guidelines for the management of acute myocardial infarction in patients presenting with ST-segment elevation. Eur Heart J. 2018:39(2):119-77.

24. O'Gara PT, Kushner FG, Ascheim DD, Casey DE, Chung MK, De Lemos JA, Ettinger SM, Fang JC, Fesmire FM, Franklin BA, et al. 2013 ACCF/AHA guideline for the management of ST-elevation myocardial infarction: a report of the American College of Cardiology Foundation/American Heart Association Task Force on Practice Guidelines. J Am Coll Cardiol. 2013;61(4):e78-140.

25. American Diabetes Association. Standards of Medical Care in Diabetes-20182. Classification and Diagnosis of Diabetes. Diabetes Care. 2018:41:S13-27.

26. Mitchell C, Rahko PS, Blauwet LA, Canaday B, Finstuen JA, Foster MC, Horton K, Ogunyankin KO, Palma RA, Velazquez EJ. Guidelines for performing a comprehensive transthoracic echocardiographic examination in adults: recommendations from the American Society of Echocardiography. J Am Soc Echocardiogr. 2019;32(1):1-64.

27. Takahashi H, Iwahashi N, Kirigaya J, Kataoka S, Minamimoto Y, Gohbara M, Abe T, Okada K, Matsuzawa Y, Konishi M, et al. Glycemic variability determined with a continuous glucose monitoring system can predict prognosis after acute coronary syndrome. Cardiovasc Diabetol. 2018;17:116.

28. Lee G, Kim SM, Choi S, Kim K, Jeong S, Son JS, Yun J, Park SM. The effect of change in fasting glucose on the risk of myocardial infarction, stroke, and all-cause mortality: a nationwide cohort study. Cardiovasc Diabetol. 2018;17:51.

29. Donagaon S, Dharmalingam M. Association between glycemic gap and adverse outcomes in critically ill patients with diabetes. Indian J Endocrinol Metab. 2018;22(2):208.

30. Worthley MI, Holmes AS, Willoughby SR, Kucia AM, Heresztyn T, Stewart S, Chirkov YY, Zeitz CJ, Horowitz JD. The deleterious effects of hyperglycemia on platelet function in diabetic patients with acute coronary syndromes mediation by superoxide production, resolution with intensive insulin administration. J Am Coll Cardiol. 2007:49(3):304-10.

31. Capes SE, Hunt D, Malmberg K, Gerstein HC. Stress hyperglycaemia and increased risk of death after myocardial infarction in patients with and without diabetes: a systematic overview. Lancet. 2000;355(9206):773-8.

32. Ishihara M, Kojima S, Sakamoto T, Kimura K, Kosuge M, Asada Y, Tei C, Miyazaki S, Sonoda M, Tsuchihashi K, et al. Comparison of blood glucose values on admission for acute myocardial infarction in patients with versus without diabetes mellitus. Am J Cardiol. 2009;104(6):769-74.

33. Teraguchi I, Imanishi T, Ozaki Y, Tanimoto T, Kitabata H, Ino Y, Ishibashi K, Komukai K, Hirata K, Akasaka T. Impact of stress hyperglycemia on myocardial salvage following successfully recanalized primary acute myocardial infarction. Circ J. 2012;76(11):2690-6.

34. Marenzi G, Cosentino N, Milazzo V, Metrio MD, Bartorelli AL. Prognostic value of the acute-to-chronic glycemic ratio at admission in acute myocardial infarction: a prospective study. Diabetes Care. 2018;41(4):C171732

35. Liao W, Wang J, Lin C, Yang C, Hsu C, Chu S, Chu C, Tsai S. Elevated glycemic gap predicts acute respiratory failure and in-hospital mortality in acute heart failure patients with diabetes. Sci Rep. 2019;9(1):1-9.

36. Lee TF, Burt MG, Heilbronn LK, Mangoni AA, Wong VW, McLean M, Cheung NW. Relative hyperglycemia is associated with complications following an acute myocardial infarction: a post hoc analysis of $\mathrm{HI}-5$ data. Cardiovasc Diabetol. 2017;16(1):157.

\section{Publisher's Note}

Springer Nature remains neutral with regard to jurisdictional claims in published maps and institutional affiliations. 